\title{
Update on the Use of Synthetic Mesh in Reconstructive and Cosmetic Breast Surgery
}

\author{
Hilton Becker ${ }^{1}$
}

Published online: 5 August 2020

(C) Springer Science+Business Media, LLC, part of Springer Nature and International Society of Aesthetic Plastic Surgery 2020

\section{Introduction}

The introduction of acellular dermal matrix greatly enhanced the results of implant-based breast reconstruction. However, with the exorbitant cost, and increased infection rate, I began looking at the use of synthetic mesh as an alternative.

There were numerous meshes available that were designed for hernia and abdominal wall repair.

I initially used Polypropylene mesh to anchor the pectoralis muscle back into position after release. Although it functioned well, it lacked elasticity and it was too rigid, resulting in flattening of the lower pole of the breast. I then used Ultrapro mesh (Ethicon) polypropylene/polyglactin which is a combination mesh consisting of proline and vicryl. When the vicryl absorbs a finer prolene mesh remains, however, it was still too rigid, I, therefore, looked at absorbable meshes. Vicryl mesh (Ethicon) polyglactin, knitted or woven, functioned well initially but absorbed rapidly leaving no residual support.

Tiger mesh [1] is a copolymer of glycolide lattice and trimethylene carbonate, a combination mesh consisting of two long-term absorbable meshes offered the advantage of a soft flexible mesh offering excellent initial strength which becomes more flexible as the shorter-term mesh absorbs. The residual long-term mesh induces collagen neogenesis; however, tiger mesh is braided and therefore more prone to infection.

\section{Hilton Becker}

Hilton@beckermd.com

1 Hilton Becker MD Clinic of Plastic Surgery, Boca Raton, USA
I, therefore, resorted to a monofilament mesh. The only monofilament finer meshes available were both permanent, Tiloop (Titanium-coated polypropylene) and Restorelle [2] (Ultrafine polypropylene). They both functioned well but were still too rigid. There was also the concern of leaving a permanent mesh within the breast. With the introduction of Phasix and Galaflex (poly-4-hydroxybutyrate), [3] both long-term absorbable monofilament meshes, they became my mesh of choice. However, when I began pre-pectoral breast reconstruction, I found this mesh to be too bulky and rigid.

None of these meshes were specifically developed and designed for breast surgery.

A new mesh which has specific elements of its design that enhance outcomes in breast surgery, Durasorb (polydioxanone), [4] has recently been released. It is a monofilament, long-term absorbable mesh which is fine and flexible and suitable for all aspect of breast surgery. Initial results are most encouraging.

In the future, I believe we will see a finer mono-filament three-dimensional, long-term absorbable mesh. Nanofibers of permanent material could be incorporated, which will simulate the scaffold of naturally occurring connective tissue.

\section{References}

1. Becker H, Lind JG (2013) The use of synthetic mesh in reconstructive, revision, and cosmetic breast surgery. Aesthet Plast Surg 37:914-921

2. Alinsod AM, Patel MP, Erickson TB (2012) Durability and complications of an ultra lightweight transvaginal mesh in the 
treatment of pelvic organ prolapse. J Min Invasive Gynecol 19(6): $\mathrm{s} 32-33$

3. Williams SF, Martin DP, Moses AC (2016) The history of GalaFLEX P4HB Scaffold. Aesthet Surg J 36(Suppl 2):S33-S42

4. Mlodinow AS, Kuzycz M, Hasse M, Cruikshank T, Wiegel SW, Ellis MF, Kim JYS (2019) Biomechanical and histologic evaluation of a novel absorbable mesh in a porcine model of abdominal wall repair. In: Poster presented at plastic surgery research council. May 2-5, 2019, Baltimore, MD

Publisher's Note Springer Nature remains neutral with regard to jurisdictional claims in published maps and institutional affiliations. 